\title{
Capture and Exchange of Component Information Using EXPRESS Oriented Standards
}

\author{
M. Balzer, J. Bröking, W. Wilkes \\ University of Hagen, D-58084 Hagen, Germany \\ e-mail: [first name]"."[last name]"@fernuni-hagen.de"
}

\begin{abstract}
The exchange of information about components which are built into higher level products is of utmost importance for both component manufacturers and component users. The ESPRIT project CIREP was set up to address the needs of industry for standardised procedures for defining the characteristics of components and for transmitting the information from manufacturers down to the corporate CAD databases of end users. This paper focus on two aspects of the CIREP project: (1) The CIREP datamodel which is based on the EXPRESS models of the standards IEC61360 and ISO13584 (PLIB), and (2) the implementation of a capture tool for component data which has been implemented using an EXPRESS database and a software development environment for EXPRESS called EXPRESS+/SDAI+.
\end{abstract}

Keywords

Standard models for component information, Product and process interchange, ISO13584, PLIB, IEC61360, STEP, EXPRESS

This work was supported by the European Union through the ESPRIT project 22124 (CIREP) 


\section{INTRODUCTION}

In almost all cases products are not produced from scratch but they are designed and manufactured by use of components which are bought from component manufacturers. Components may be very elementary like nuts and bolts in the mechanical area or capacitors in the electronic area, but they may also be very complex, as for instance complete microprocessors. For the "consumer" of components it is of utmost importance to get early and reliable information on the components supplied by a manufacturer: He needs this information for the selection of appropriate components and for the design of higher level products which incorporate these components.

To facilitate the information flow, manufacturers and users have to agree on a common representation of the component information and on a defined meaning of data. For this purpose, standards have been developed for a common representation of component information. The IEC61360 standards provides an EXPRESS model which describes a dictionary of component characteristics (or properties) and which allows the classification of components in a classification tree. IEC61360 Part 4 contains a dictionary for electrotechnical components described according to this model. ISO13584 (PLIB) extends the model of IEC61360 by supporting the representation of actual component information compliant with the dictionary model.

This paper describes the CIREP project which uses these standards to support the exchange of component information. It has slightly extended the models defined by the standards and it has developed tools to deal with data as define in IEC61360 and in PLIB. The following section gives a brief overview of the project. Section 3 describes the models provided by the standards and the extensions added by CIREP, and finally, section 4 discusses some aspects of the CIREP tool CIMT/CICT which allows to handle component information according to the standards.

\section{OVERVIEW OF CIREP}

In the Esprit project CIREP (Component Information REPresentation), manufacturers (AVX (GB), Philips (NL), Siemens (D)), component users (Aerospatiale $(\mathrm{F})$, Thomson CSF $(\mathrm{F})$ ), and technology providers (Codus (GB), Euritis (F), Spring (F), University of Hagen (D)) collaborate to design and implement a system which supports the exchange of component data between manufacturers and component users. The component information is transferred in electronic form via the Internet or via point-to-point connections. The component manufacturers store the component information decentralised and autonomously on their own servers. Information brokers may facilitate the distribution of information and allow an integrated search across several manufacturers. The 
component user can search for information either in the manufacturers' databases or in the database of an information broker to identify relevant components and to download information available about them. The downloaded information can then be integrated into the corporate design database of the end user to make it available for the design of higher level products.

CIREP has been working on the following areas:

- The CIREP methodology describes the way how information flows from the manufacturers to the end users and the kind of interactions which occur among the various actors. Actors in this methodology are manufacturers and users, but also standards organisations which create and maintain standardised dictionaries, and brokers which add value to the component information (e.g. by adding simulation models or by supporting manufacturer independent search). Specific emphasis has been put on security and IPR protection. Manufacturers provide much information for free, but part of the information can be confidential, and in particular the brokers rely on IPR protection since they earn their money with selling information - not with selling real components. For more information see Wilkes, et.al. (1998).

- The CIREP data model basically adopts the standards IEC61360 (IEC 61360-2 (1997)) and PLIB (ISO/CD 13584-24.2 (1997)). These standards are based on a common meta model defined in EXPRESS (ISO 10303-11 (1994)). CIREP has extended the models by the concept of CDILs (Component Class Data Item Lists). CDILs support the handling of component data by specifying the way in which this data is organised. In section 3 , an overview of the models of IEC61360 and PLIB and the CIREP extensions is given.

- The CIREP tools support the CIREP methodology and deal with the CIREP data model. CIREP has developed tools for data capture (component information modelling and capture tool, CIMT/CICT), for searching and downloading information from a database, for customising the data to enable an integration with the corporate database of an end user (Component Information Handling System, CIHS), and for ensuring security of data and protection of IPRs. In this paper, we describe the CIMT/CICT tool in more detail which is based on a high level programming interface defined alongside the EXPRESS model (EXPRESS+/SDAI+, see section 4).

\section{CIREP DATA MODEL}

\subsection{Brief Overview of IEC61360 and ISO13584 (PLIB)} 4

IEC61360 and PLIB are based on a common meta model which is defined in EXPRESS. The overall structure of a database based on their models is sketched in figure 1. IEC61360/2 describes the meta model for a dictionary of component classes, and one instantiation is specified in IEC61360/4 which defines data 
element types and a hierarchy of electrotechnical component classes (IEC dictionary). Other manufacturer-specific dictionaries are possible as well as manufacturer-specific extensions of the standard classification. ISO13584/24 specifies a meta model for component families, and an instantiation of this meta model describes specific component families. A component family is a set of components which belong to a single component class of the dictionary. The components comprised in a family have several properties in common (e.g. the family of the 486 processors).

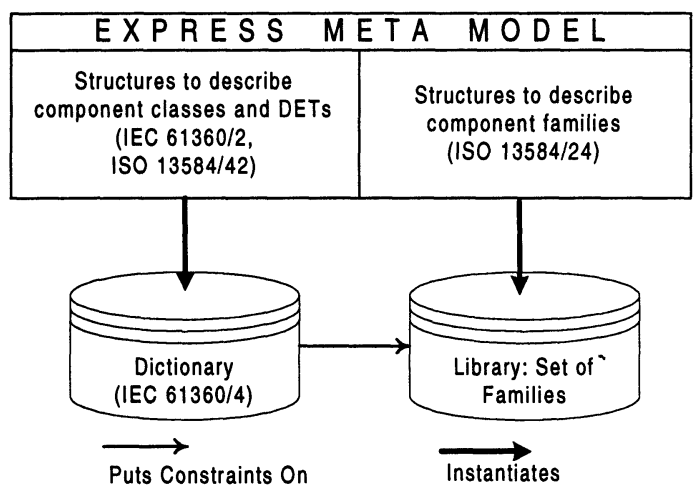

Figure 1: Overall structure of a component database based on IEC61360 / ISO13584.

The main goal of IEC61360 is the unique representation of data element types describing the characteristics of components. A data element type (DET) is described by a set of attributes like identifying attributes, semantic attributes describing the meaning of the DET, value attributes describing the way in which values for the specific characteristic are represented and measured, and relationship attributes associating the DET to component classes, to DET classes, or to conditions for the measurement of the specific characteristic.

To organise the DETs properly, IEC uses a class hierarchy where data element types are attached to classes and inherited by subclasses. This hierarchy allows to classify the components by locating general classes near the top of the hierarchy and specialising them when going down the hierarchy. A class inherits all DETs from the relevant classes above it. More information can be found in Radley(1997).

Whereas IEC61360 describes data element types and component classes, PLIB specifies the representation of component families. According to PLIB, a family is described intentionally, i.e. instead of listing all components with all their property values, the family description factors out the common property values. Furthermore, it allows to specify that property values are dependent on other 
properties thus ending up in an efficient and compact description of large sets of components.

The basic feature to specify the elements of a family set is the domain restriction. It specifies possible values for some properties defined in the corresponding class. These values may be defined e.g. by means of expressions, by the definition of ranges, or by means of (relational) tables. Figure 2 sketches an example of the kind of structures which can be defined with PLIB: The classes are organised in a class hierarchy, and properties have been attached to them. To each class at most one family is associated which groups or classifies the properties defined in the class (indicated as (G/C) in figure 2) and specifies the set of components which belong to that class by means of domain restrictions.

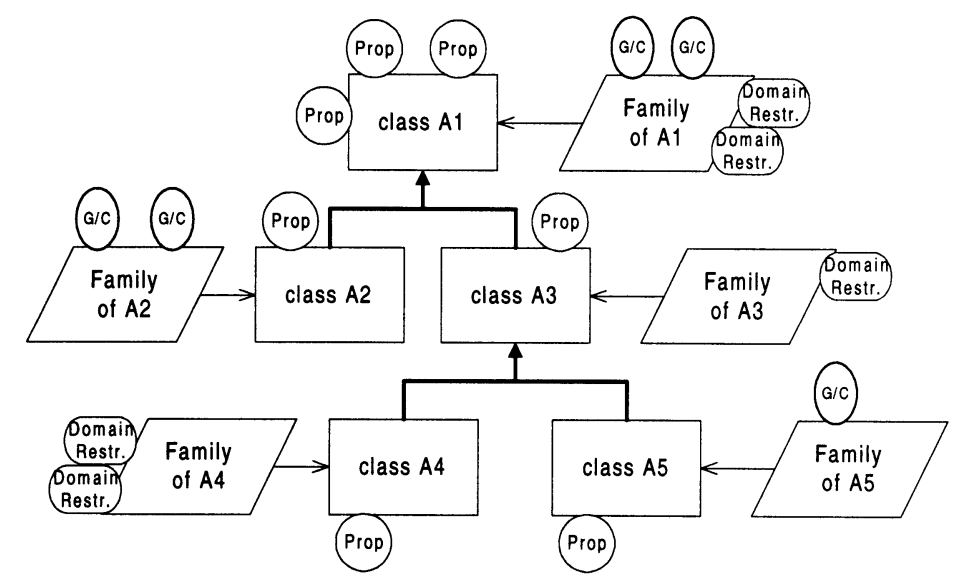

Figure 2: Class hierarchy with associated families (PLIB)

\subsection{Extensions of CIREP}

The family description of PLIB resembles the way how component families are nowadays described in data books. But a family is like a self-extracting file: The end user does not know which kind of data and data organisation the manufacturer has used for a specific family. This makes it more difficult for end users to apply automatic processes to arbitrary families as e.g. a mapping into his design database. In addition, to be integrated into the design data, the received data needs to be reliable, i.e. there should be possibilities to check the correctness of data. Another problem for CIREP is the restriction to a single family per class. If a manufacturer provides more than one family for a given class or a broker is offering several families of different manufacturers, this restricts them to belonging to different classes.

Therefore, CIREP has extended PLIB by introducing the concept of CDIL (Component Class Data Item List) which is basically a "family template" giving 
more detailed information about the structure of families. A CDIL describes in which way families are specified: Which properties are common for the respective families, which properties are dependent from others, etc. This creates a template which can be filled in for the definition of concrete families, e.g. by specifying values of the common properties or by providing functions for the derivation of dependent properties. If a family belongs to a known CDIL, it is possible to check the integrity of the family against the CDIL and to deal with it in a more standardised way. CDILs are instantiated by CDIL instances which are semantically equivalent to ISO families. More than one CDIL instance may belong to a CDIL thus enabling the storage and transfer of several CDIL instances (representing families) which all may belong to a single class.

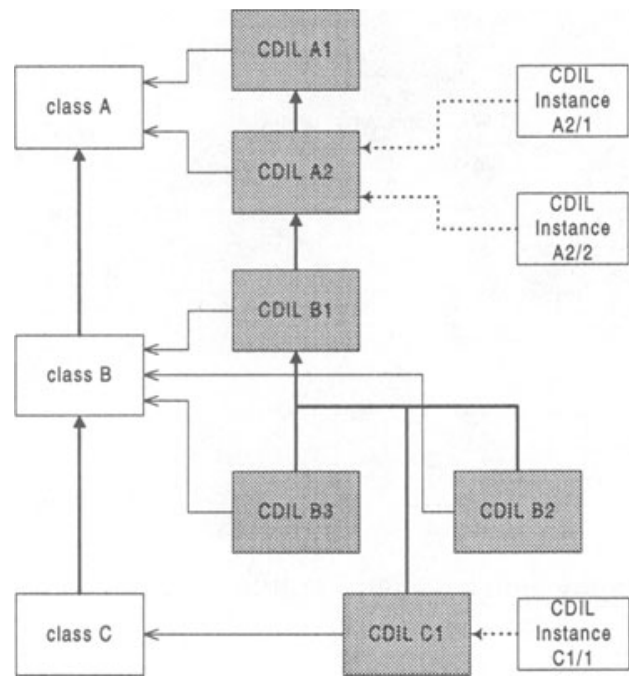

Figure 3: Example of hierarchical structures of classes and CDILs

The CDIL prescribes the structure of its CDIL instances in a number of ways :

- it provides segmentation of the class, grouping related properties together

- it can add additional attributes to each property to govern local requirements such as searchability and segmentation

- it allows the specification of table structures which are filled in for the associated CDIL instances to intentionally describe their sets of components.

A CDIL is related to a single class, and more than one CDIL may be related to the same class (see figure 3: CDIL A1 and CDIL A2). CDILs may be organised in a hierarchy, i.e. a CDIL may have a "super-CDIL". The super-CDIL is related either to the same class as the sub-CDIL or to a (transitive) superclass of the subCDIL. In figure 3, for example, CDIL B1 is the super-CDIL of B2 and B3, and all 
three CDILs are related to the same class B. B1 is also the super-CDIL of $\mathrm{C} 1$ which belongs to class $\mathrm{C}$, a subclass of B1's class $\mathrm{B}$.

\subsection{Mapping of CDILs to PLIB}

In principle, a CDIL is a dictionary element which describes the structure of its families. On the other hand, it uses the same mechanisms as a family to define the family structure (i.e. it groups the properties, it defines dependencies between properties, it defines table structures, etc.).

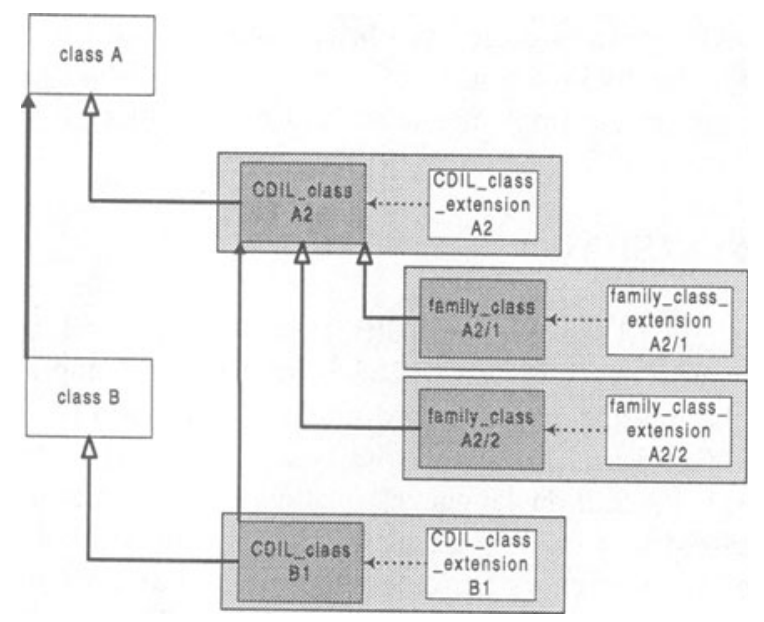

Figure 4: Representation of CDILs in the CIREP model

Therefore, CDILs are represented in the CIREP model by two PLIB entities (see figure 4): by a class and a class_extension. The CDIL_class is used to link the CDIL to a classification hierarchy by means of the is_case_of relationship provided by PLIB. An entity class_extension is normally used for specifying families; here we use the subtype cdil_class_extension for defining the family template which specifies the allowed structure of the associated CDIL instances. In the same way, a CDIL instance representing a family is mapped to a class and a class_extension: The family_class is related to a CDIL-class, again by using the is_case_of relationship, and the family_class_extension contains the actual family information. Its relationship to the template in the associated cdil_class_extension is described by means of constraints which specify how a concrete family can be defined on the basis of a given template. Figure 4 illustrates how (part of) the structure given in figure 3 is represented according to this scheme. 
As described in the CIREP overview (section 2), CIREP has developed various tools. In this paper we describe only one of them: CIMT/CICT (Component Information Modelling Tool / Component Information Capture Tool) serves as the capture tool for dictionary data and component information. It keeps its data in an EXPRESS database which can be accessed via the Internet. For the implementation of CIMT/CICT we have used our EXPRESS+/SDAI+ environment which supports the definition of higher level interfaces for EXPRESS databases. In the following, we first describe the underlying concepts of EXPRESS+/SDAIt, then we give a brief overview of the architecture of CIMT/CICT, and finally, we point out how the EXPRESS+/SDAI+ system is being used to support the implementation and enhancement of the CIMT/CICT prototype.

\subsection{EXPRESS+ / SDAI+}

Both models of IEC61360 and of PLIB have been defined by means of the modelling language EXPRESS. EXPRESS is the basic building block of STEP, a programme of the International Standardisation Organisation (ISO) to support the exchange of product data. EXPRESS (ISO 10303-11 (1994)) can be characterised as a semantic data definition language: It allows the definition of entity types, which are organised in a sub-/supertype hierarchy, it provides a rich set of basic types for the definition of entity attributes, and local constraints and global rules to further specify entities and their relationships. But EXPRESS is a pure data specification language, it does not imply any operational semantics.

To make EXPRESS models operational, STEP has introduced the Step Data Access Interface (SDAI, ISO 10303-22 (1994)) as a standardized way of accessing EXPRESS data. But SDAI provides only elementary operations like retrieval or update of attributes, deletion or creation of objects and checking of specific constraints. Thus, it is the task of application programs to produce higher level and domain specific functionality on the basis of the simple SDAI functions.

To improve this situation, we have extended EXPRESS to EXPRESS+ (Balzer and Bröking (1996), Kretzberg and Wilkes (1996a), (1996b) ). Basically, EXPRESS+ extends EXPRESS by entity methods, i.e. operations which are properties of entities in the same way as attributes. Thus, EXPRESS+ makes EXPRESS truly object-oriented. Both the signature and the body of a method are coded with the syntax constructs provided by EXPRESS.

Additionally, EXPRESS+ provides mechanisms which allow the definition of higher concepts. Of particular importance are the explicit definition of relationships and relationship templates. Relationship templates are a key concept for designing sound and consistent object oriented models since they perform a systematic migration of relationship specific structures and methods into the 
involved entities. Another important concept is the linking of constraints and methods which supports the application programmer in writing code of better quality which satisfies the constraints defined in the model.

The methods defined in EXPRESS+ are provided to applications by means of a programming interface called SDAI+. SDAI+ is an extension of SDAI; the most important extension is the function do_method which allows the call of EXPRESS+ methods from an application program. The SDAI+ system executes the method: First, the dispatcher checks the validity of the method call before the associated code contained in the method library is executed. The SDAI+ system encapsulates SDAI which is used as the basic data access mechanism. The method library and some model specific parts of the dispatcher are automatically generated by the SDAI+ generator (see figure 5).

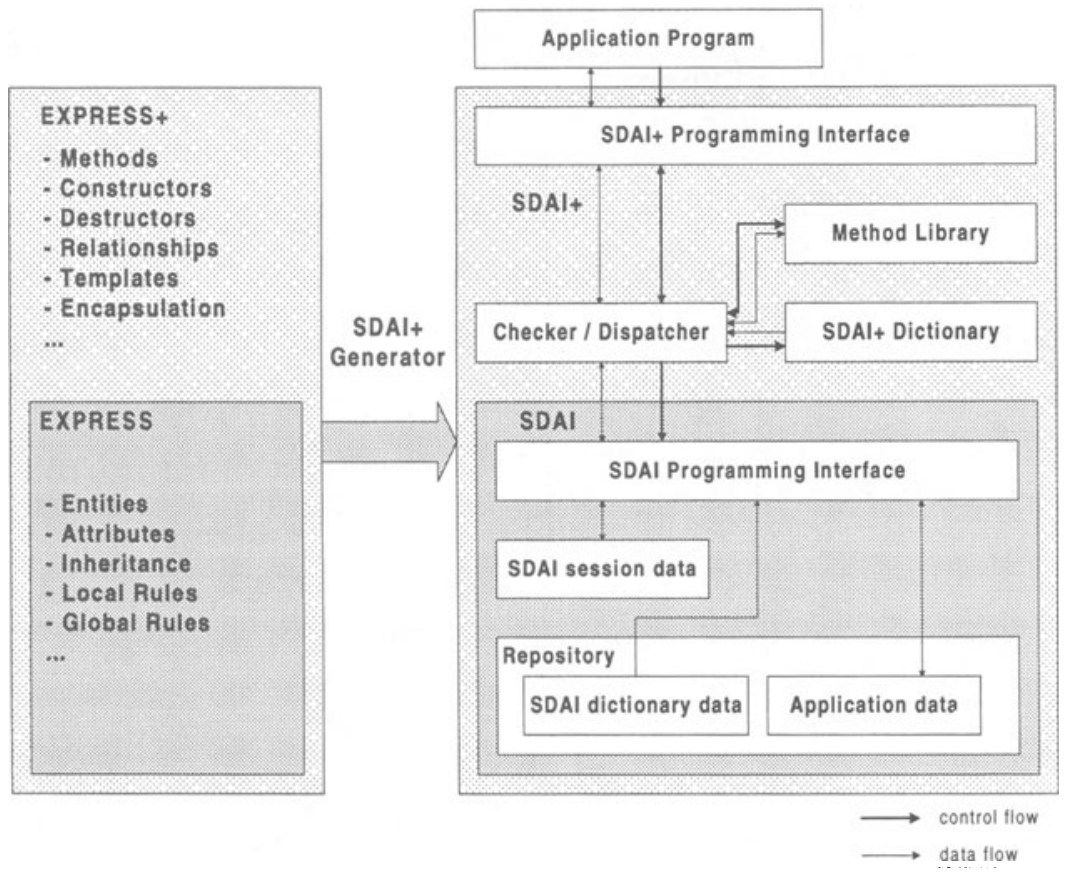

Figure 5: Architecture of the EXPRESS+/SDAI+ system

\subsection{CIMT/CICT Architecture}

CIMT/CICT has been implemented with Java as a client-server application. The architecture is sketched in figure 6. The client communicates with the server via the Java RMI (Remote Method Invocation) which is a CORBA-like service. The server handles the requests sent by the client by using an EXPRESS database which provides an SDAI interface. The SDAI is encapsulated by a (partially) generated SDAI+. For the implementation of the capture tool, the models of 
IEC61360 and PLIB have been extended by appropriate templates and methods in EXPRESS+, and the generated SDAI+ provide high level operations for dealing with complex structures like CDILs, families, etc. These database objects are translated into JAVA objects to be transferred to the client.

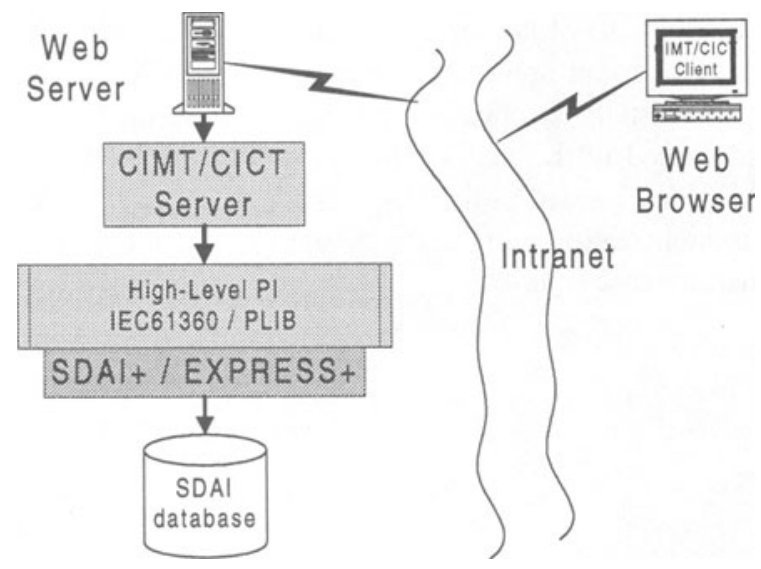

Figure 6: Architecture of CIMT/CICT

\subsection{Use of EXPRESS+/SDAI+ to implement CIMT/CICT}

The ISO13584 High Level Programming Interface (see figure 6) realises the common functions which are needed to deal with ISO13584 dictionary data (i.e. creation of classes, properties, CDILs, management of relationships etc.) in close association to the underlying data model. Operations are defined as object oriented methods attached to the existing ISO13584 entities.

Using this approach of defining data management operations using EXPRESS+, a great deal of the effort normally needed to implement a high level PI for an EXPRESS model can be avoided, as managing EXPRESS data is most easy from within the EXPRESS environment itself. The equivalent for any function of the HLPI written in $\mathrm{C} / \mathrm{C}++$ typically needs about five times as many lines of code and is of course far less readable and maintainable.

In particular, the use of EXPRESS+ supports the realisation of the following aspects of the ISO13584 High Level Programming Interface:

- Methods to support the repeated access to complex structures. There are many objects and relations defined by ISO13584 which are similar in structure and handling, e.g. the version control system and the naming of dictionary elements. The EXPRESS+ language provides means to handle these parts in a generic way, by methods defined on super-type or template level which eliminates the need to write specific code for each single case. 
- Methods for exporting sensible pieces of data. Data is exchanged from CIMT/CICT to other CIREP tools in form of a STEP Physical File (ISO 1303 Part 21). To collect the data which belongs to sensible items (e.g. a class, a CDIL, a family) requires to establish and traverse the specific semantic relationships of the ISO13584 model. This is most convenient on the level of the data model itself rather than using low level interface functions of SDAI.

- Dealing with PLIB specialities in a flexible and efficient way. For instance, PLIB uses a specific mechanism to relate elements of the dictionary to other entities. By the use of relationships and relationship templates inn EXPRESS+, the implementation of this mechanism can be adapted to the access requirements in the database. Simultaneously, the export of data into the Physical File Format can (re-)generate valid PLIB compliant data for data exchange. All this is hidden from the application programmer who only gets abstract navigation and manipulation operation to deal with these relationships.

- Supporting consistency of data: A STEP database normally does not do constraint checking automatically on every update, but requires an explicit check request to be triggered by the application. By accessing STEP objects through their specific object encapsulation interface, the object methods can provide a direct checking of constraints where appropriate. On the level of families, the family data can be easily checked for compliance with the actual dictionary definition constraints, which also are modelled by ISO13584 objects.

- Execution of operations defined within an ISO13584 dictionary: ISO13584 offers means to define operations, in particular relational table operations and table queries within its model. A function is modelled as a sequence of statements and expressions which are stored in form of a syntax tree. As all syntax tree elements are instances of ISO13584 entities representing specific expression elements (like for example an addition of two expressions), these trees can be made executable by defining the actions for each of these elements as EXPRESS+ methods attached to the entities. Then the execution of an ISO13584 function is basically just an evaluation of the action methods of the syntax tree, so no compiler or interpreter needs to be written.

\section{CONCLUSION}

In this paper we have given an overview of the work in the CIREP project. The project supports the exchange of component information based on the standards IEC61360 and PLIB. CIREP has added the concept of CDIL (Component Class Data Item List) and mapped it to PLIB. As an example of the tools developed in the project, the paper has introduced CIMT/CICT and the underlying EXPRESS+/SDAI+ environment. A first version of the integrated CIREP system has been demonstrated in July, a complete version is expected by December. 
Currently, further efforts are spent to support the interoperation of IEC61360 and PLIB with other standards for component representation, as e.g. the PCIS (Pinnacles Component Information Standard) and CIDS (Component Information Dictionary Standard) of the US-based ECIX consortium. For this purpose, CIMT/CICT is being extended to also support export and import facilities for these SGML-based representations.

\section{REFERENCES}

Balzer, M., Bröking, J. (1996): Automatic Generation of Programming Interfaces for Information Models. Diploma thesis, University of Hagen (in German).

IEC 61360-2 (1997): Common IEC/ISO dictionary schema.

ISO 10303-11 (1994): EXPRESS Language Reference Manual.

ISO 10303-21 (1994): Step Physical File Format.

ISO/CD 10303-22 (1994): Standard Data Access Interface, ISO TC184/SC4/WG7/N375.

ISO/CD 13584-24.2 (1997): Parts library: Logical resource: Logical model of supplier library.

Kretzberg, T., Wilkes, W. (1996): Extending EXPRESS by Relationship Templates: Supporting Reusable Modelling and Interface Generation for Technical Applications. In Proc. Int. Conf. On Data and Knowledge Systems for Manufacturing and Engineering (DKSME'96), Phoenix, Arizona.

Kretzberg, T., Wilkes, W. (1996): Generation of Application-Specific Programming Interfaces. In: Product Data Journal, ProSTEP Association, No. 2.

Radley, D. E. (1997): Component information standardisation. In Proc. $2^{\text {nd }}$ Workshop on Libraries, Component Modeling and Quality Assurance, Toledo, Spain.

Wilkes, W., Balzer, M, Bröking, J., Radley, D. (1998): An overview of CIREP methodology. In Proceedings European Conference Product Data Technology Days 1998, Watford, UK.

\section{BIOGRAPHIES}

Michael Balzer got a degree in Computer Science at University of Dortmund in 1996. He is currently a member of the CIREP project staff at University of Hagen.

Jens Bröking got a degree in Computer Science at University of Dortmund in 1996. He is currently a member of the CIREP project staff at University of Hagen.

Dr. Wolfgang Wilkes got his diploma degree in Computer Science from the University of Dortmund (Germany) in 1982 and his $\mathrm{PhD}$ from the University of Hagen in 1987. He is working since 1982 as research assistant and project leader at the University of Hagen. He has been involved in several ESPRIT projects in the areas of CAD databases and EXPRESS modelling and tools (Jessi Common Frame, ECIP, ESIP, CIREP, STEPWISE). 